\title{
Cytotoxicity Studies of the Crude venom and Fractions of Persian Gulf Snail (Conus textile) on Chronic Lymphocytic Leukemia and Normal Lymphocytes
}

\author{
Ahmad Salimi',2, Shayan Salehian ${ }^{3}$, Akram Aboutorabi ${ }^{4}$, Amir Vazirizadeh ${ }^{5}$, \\ Vahed Adhami², Seyyed Hossein Sajjadi Alehashem², Enayatollah Seydi,7*, Jalal \\ Pourahmad $^{3 *}$
}

\begin{abstract}
Background: Marine animals have been considered by many researchers due to their various pharmacological effects. One group of marine animals that have been studied is cone snails. The conotoxin obtained from these marine animals has various therapeutic effects. Methods: This study was designed to investigate the apoptotic effects of crude venom of Conus textile and its fractions (A and B) on chronic lymphocytic leukemia (CLL) cells. Accordingly, parameters such as cell viability, reactive oxygen species (ROS) level, collapse in mitochondrial membrane potential (MMP), lysosomal membrane damage and caspase-3 activation were evaluated. Results: The results showed that the crude venom (50, 100 and $200 \mu \mathrm{g} / \mathrm{ml})$ from Conus textile and its fraction B (50,100 and $200 \mu \mathrm{g} / \mathrm{ml})$ significantly reduced viability in CLL B-lymphocyte. In addition, exposure of CLL B-lymphocyte to fraction B $(50,100$ and $200 \mu \mathrm{g} / \mathrm{ml})$ was associated with an increase in the level of ROS, the collapse of the MMP, damage to the lysosomal membrane, and activation of caspase-3. Conclusion: According to results, it was concluded that fraction B from crude venom of Conus textile causes selective toxicity on CLL B-lymphocyte with almost no effect on a normal lymphocyte. Furthermore, this venom fraction could be a promising candidate for induction of apoptosis in patients with CLL through the mitochondrial pathway.
\end{abstract}

Keywords: Conus textile- B-Lymphocyte- mitochondria- apoptosis- oxidative stress- Persian Gulf

Asian Pac J Cancer Prev, 22 (5), 1523-1529

\section{Introduction}

Today, cancer is one of the most important health problems and the cause of human mortality in worldwide. According to the World Health Organization in 2018, cancer is responsible for the death of 9.6 million humans worldwide (Oroz-Parra et al., 2020; Sun et al., 2020). Chronic lymphocytic leukemia (CLL) is the most common type of leukemia that occurs in adults. CLL cannot be treated with traditional therapies and defects in signaling apoptosis are involved in the progression of the malignancy (Salimi et al., 2015; Teoh and Goh, 2021). Natural compounds of marine origin have been considered by many researchers as one of the important sources for drug discovery. In recent years, many of these compounds have been used in the treatment of malignancies due to their various pharmacological properties. Furthermore, animal venoms (such as cone snails) have been suggested as an alternative approach for anti-cancer therapies. Animal venoms have different pharmacological functions due to their unique bioactive molecules. Isolation of compounds with specific anticancer effects from animal venoms has become an exciting topic in cancer research (Abdel-Rahman et al., 2013; Ruiz-Torres et al., 2017; Chatterjee, 2018; Wali et al., 2019; Jimenez et al., 2020; Sukmarini, 2021).

Marine cone snails (Conus) are a large group of carnivorous predators that are distributed in tropical and subtropical water. Research has shown that there are more than 700 species of Conus around the world. Venoms isolated from cone snails have various pharmacological effects (such as anti-cancer effect). Furthermore, the venom of these marine animals contains a rich source of a peptide called conotoxins. Conotoxins have been reported

${ }^{1}$ Traditional Medicine and Hydrotherapy Research Center, Ardabil University of Medical Sciences, Ardabil, Iran. ${ }^{2}$ Department of Pharmacology and Toxicology, School of Pharmacy, Ardabil University of Medical Sciences, Ardabil, Iran. ${ }^{3}$ Department of Pharmacology and Toxicology, Faculty of Pharmacy, Shahid Beheshti University of Medical Sciences, Tehran, Iran. ${ }^{4}$ Halal Research Center of Islamic Republic of Iran, Tehran, Iran. ${ }^{5}$ Persian Gulf Research Institute, Marine Biology and Fishery Sciences Department, Persian Gulf University, Iran. ${ }^{6}$ Department of Occupational Health and Safety Engineering, School of Health, Alborz University of Medical Sciences, Karaj, Iran. ${ }^{7}$ Research Center for Health, Safety and Environment, Alborz University of Medical Sciences, Karaj, Iran.*For Correspondence: enayat.seydi@yahoo.com, j.pourahmadjaktaji@utoronto.ca 
to have several pharmacological and physiological activities (Gao et al., 2017a; Gao et al., 2017b; Fu et al., 2018; Alburae and Mohammed, 2020; Oroz-Parra et al., 2020).

In recent years, mitochondria have been considered by many researchers as a therapeutic target. This organelle is involved in the generation of reactive oxygen species (ROS) and energy and in signaling cell death. Research has shown that cancer cells are vulnerable to a rise in ROS generation and subsequent oxidative stress. Also, a rise in the level of ROS can be associated with the induction of cell death signaling. Therefore, an increase in the level of ROS can kill cancer cells (Seydi et al., 2016; Seydi et al., 2018). Our previous study showed that crude venom of Conus textile has the ability to increase ROS and induction of apoptosis signaling in U87MG human glioma cells through the mitochondrial pathway (Salimi et al., 2020). This research was aimed to investigate the apoptotic effects of crude venom of Conus textile and its fractions (A and $\mathrm{B}$ ) on CLL.

\section{Materials and Methods}

\section{Chemicals}

2, 7-dicloroflurescein diacetate (DCHF-DA), rhodamine 123 (Rh 123) and acridine orange were purchased from Sigma-Aldrich Chemical Co. (St. Louis, MO, USA). Furthermore, caspase-3 assay kit were purchased from Sigma-Aldrich Chemical Co. (Taufkirchen, Germany). All other chemicals were of the highest commercial grade available.

\section{Collection and extraction of venom}

The Conus textile samples were collected from Larak Island in the Persian Gulf, in the south of Iran. The stages of toxin extraction are described in our previous study (Salimi et al., 2020).

\section{Chromatography Methods}

Lyophilized Conus textile venom (100 mg) was dissolved in $2 \mathrm{~mL}$ of $0.2 \mathrm{M}$ ammonium acetate buffer. Venom fractions were separated by using a Sephadex $G$ 50 column $(2 \times 150 \mathrm{~cm})$ previously equilibrated and eluted with $0.2 \mathrm{M}$ ammonium acetate buffer ( $\mathrm{pH} 7.5)$. And were detected at $280 \mathrm{~nm}$ (Rigby et al., 1999). Finally, fractions were lyophilized and kept at $4^{\circ} \mathrm{C}$ for further evaluation.

\section{CLL sample and B-lymphocyte isolation}

In this study, samples were collected from 10 patients with CLL. All experiments were performed based on standard protocols approved by the ethic of Shahid Beheshti University of Medical Sciences, Tehran, Iran (ethic number: IR.SBMU.PHARMACY.REC.1398.315). Furthermore, all the CLL patients and healthy donors signed an informed consent form. Subsequently, B- lymphocytes were isolated from the blood of samples (obtained from the CLL patient and healthy donors) using Ficoll-Paque and differential centrifugation (Salimi et al., 2015).

\section{Cell viability assay}

To perform this test, CLL B- lymphocytes and normal B- lymphocytes were placed in 96-well plates with RPMI 1640 culture medium (104 cells per well). Then, both groups were treated to crude venom and fraction $\mathrm{A}$ and $\mathrm{B}$ from Conus textile at concentrations of 1, 5, 10, 50, 100 and $200 \mu \mathrm{g} / \mathrm{ml}$ for 12 hours. In the next step, MTT $(0.5 \mathrm{mg} / \mathrm{ml})$ was added to both groups for 4 hours. Then, DMSO was added to dissolve the formazone crystals. Finally, the absorbance of the samples was read using a spectrophotometer at $570 \mathrm{~nm}$ for cell viability assay (Zhang et al., 2008).

\section{ROS assay}

At first, CLL B- lymphocytes were placed in 24-well plates with RPMI 1640 culture medium $\left(10^{4}\right.$ cells per well). Then, CLL B- lymphocytes were treated to fraction $B$ from crude venom of Conus textile (50, 100 and 200 $\mu \mathrm{g} / \mathrm{ml})$. PBS was used to wash CLL B- lymphocytes. In addition, CLL B- lymphocytes were incubated with DCFH-DA reagent and centrifuged for 1 minute at 1,000 $\mathrm{rpm}$. In the following, we removed the DCFH-DA reagent and re-washed the cells with PBS. Finally, fluorescence intensity (DCF) was evaluated using flow cytometry (Cyflow Space-Partec, Germany) and the mean of fluorescence intensity was analyzed by software (FlowJo) (Salimi et al., 2020).

Mitochondrial Membrane Potential (MMP) collapse assay Briefly, CLL B- lymphocytes were placed in 24-well plates with RPMI 1640 culture medium (104 cells per well). Then, CLL B- lymphocytes were treated to fraction $B$ from crude venom of Conus textile (50, 100 and 200 $\mu \mathrm{g} / \mathrm{ml})$. PBS was used to wash CLL B- lymphocytes. In addition, CLL B- lymphocytes were incubated with Rh123 reagent and centrifuged for 1 minute at 1,000 rpm. In the following, we removed the Rh123 reagent and re-washed the cells with PBS. Finally, fluorescence intensity (Rh123) was evaluated using flow cytometry (Cyflow Space-Partec, Germany) and the mean of fluorescence intensity was analyzed by software (FlowJo) (Baracca et al., 2003).

\section{Evaluation of lysosomal damage}

At first, CLL B- lymphocytes were placed in 24-well plates with RPMI 1640 culture medium $\left(10^{4}\right.$ cells per well). Then, CLL B- lymphocytes were treated to fraction B from crude venom of Conus textile (50, 100 and 200 $\mu \mathrm{g} / \mathrm{ml})$. PBS was used to wash CLL B- lymphocytes. In addition, CLL B- lymphocytes were incubated with acridine orange reagent and centrifuged for 1 minute at $1,000 \mathrm{rpm}$. In the following, we removed the acridine orange reagent and re-washed the cells with PBS. Finally, fluorescence intensity (acridine orange) was evaluated using flow cytometry (Cyflow Space-Partec, Germany) and the mean of fluorescence intensity was analyzed by software (FlowJo) (Brunk et al., 1995).

\section{Caspase-3 activity assay}

Caspase- 3 activity was assayed using the Sigma Caspase-3 assay kit (Sigma-Aldrich, Taufkirchen, 
Germany), and the concentration of the p-nitroaniline released from the substrate at $405 \mathrm{~nm}$ used for caspase-3 activity was assayed.

\section{Statistical analysis}

Results are showed as means \pm SD. All statistical analyses were done using the GraphPad Prism software, version 6. All experiments were analyzed using one-way analysis of variance (ANOVA) followed by the Tukey test. Statistical significance was set at $\mathrm{P}<0.05$.

\section{Results}

\section{Fractionation of Conus textile toxins}

In order to obtain active molecules crude venom of Conus textile, was used Sephadex G50 gel filtration. The column was equilibrated and eluted with ammonium acetate buffer $(0.2 \mathrm{M})$. As shown in Figure 1, two main peaks were collected from a Sephadex G-50 column, for further evaluations.

Conus textile (crude venom and fraction $B$ ) decreased cell viability in CLL B- lymphocytes

After 12 hours of treatment, the results showed that crude venom of Conus textile at concentrations of 50,100 and $200 \mu \mathrm{g} / \mathrm{ml}$ decreased cell viability in CLL B-lymphocytes (Figure 2A). Next, the effects of fractions $\mathrm{A}$ and $\mathrm{B}$ (from crude venom of Conus textile) on cell viability in normal B-lymphocytes and CLL B-lymphocytes were investigated. As shown in Figure $2 \mathrm{~B}$, fraction $\mathrm{A}$ was not able to reduce cell viability in CLL cells. However, fraction $B$ at concentrations of 50,100 and $200 \mu \mathrm{g} / \mathrm{ml}$ and after 12 hours of treatment was able to reduce cell viability in CLL B-lymphocytes (Figure $2 \mathrm{C}$ ). Furthermore, this effect of fraction B has not been observed on normal B-lymphocytes (Figure 2D). In the following, the effects of fraction $\mathrm{B}$ on the desired parameters were investigated.

Fraction $B$ increased ROS generation in CLL Blymphocytes

To assess the level of ROS generation, CLL
B-lymphocytes were treated to different concentrations of fraction B from crude venom of Conus textile. Using flow cytometry, the results showed that fraction B at concentrations of 50,100 and $200 \mu \mathrm{g} / \mathrm{ml}$ was able to increase the level of ROS in CLL B- lymphocytes (Figure 3). This effect of fraction $\mathrm{B}$ has not been observed on normal B-lymphocytes (data not shown.). Compounds that have the ability to increase ROS generation can help kill cancer cells.

Fraction B induced MMP collapse in CLL B-lymphocytes

The collapse in the MMP is considered to be one of the most important events in cell death. Flow cytometry results showed that fraction $\mathrm{B}$ at all concentrations (50, 100 and $200 \mu \mathrm{g} / \mathrm{ml}$ ) used compared to the control group caused the collapse of the MMP in CLL B-lymphocytes (Figure 4).

Fraction $B$ induced lysosomal damage in CLL Blymphocytes

Flow cytometry results showed that incubation of CLL B-lymphocytes with fraction B at concentrations of 50,100 and $200 \mu \mathrm{g} / \mathrm{ml}$ significantly $(\mathrm{p}<0.05)$ leads to lysosomal damage in the compared to the control group (Figure 5). This effect of fraction B has not been observed on normal cells (data not shown.).

Fraction $B$ increase caspase-3 activity in CLL Blymphocytes

In Figure 6, caspase-3 activity in a dose-dependent pattern was significantly increased in CLL B-lymphocytes compared to the control group.

\section{Discussion}

In the present study, the toxicity effects of isolated fractions of Persian Gulf snail crude venom (Conus textile) on CLL B-lymphocytes were investigated. In recent years, according to data from the WHO the number of new cancer cases is on the rise. Cancer treatment is still considered a medical challenge worldwide. Therefore,

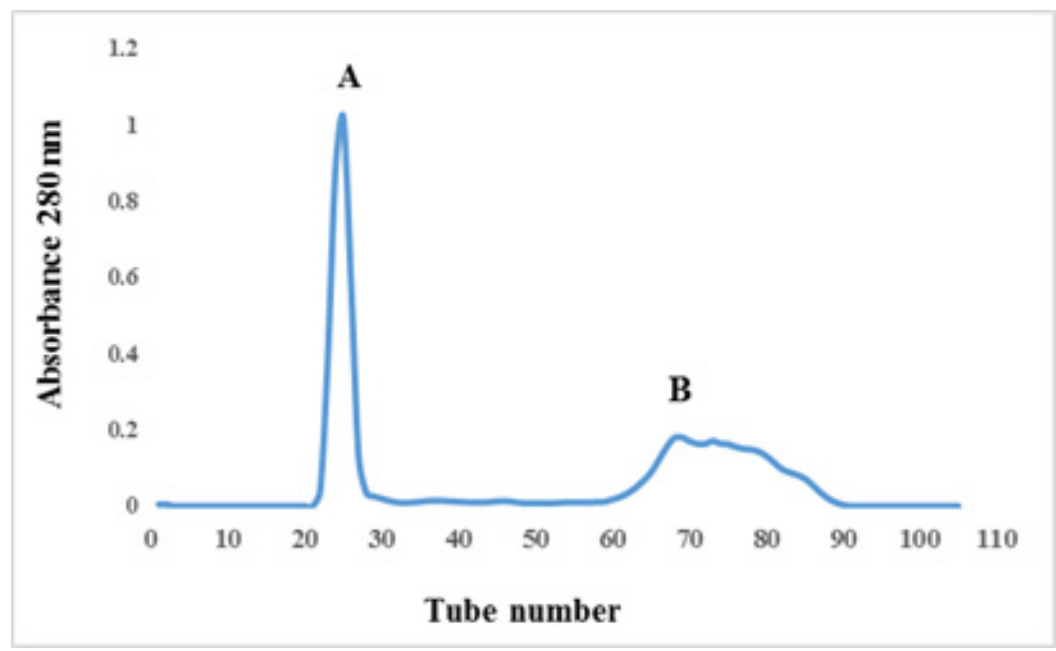

Figure 1. Gel Filtration Diagram of Conus Textile Crude Venom (100 mg), Dissolved Venom in Ammonium Acetate Buffer and Loaded on a Sephadex G50 Column, Previously Equilibrated and Eluted with 0.2 M Ammonium Acetate, $\mathrm{pH} 7.5$, and Detected at $280 \mathrm{~nm}$ 


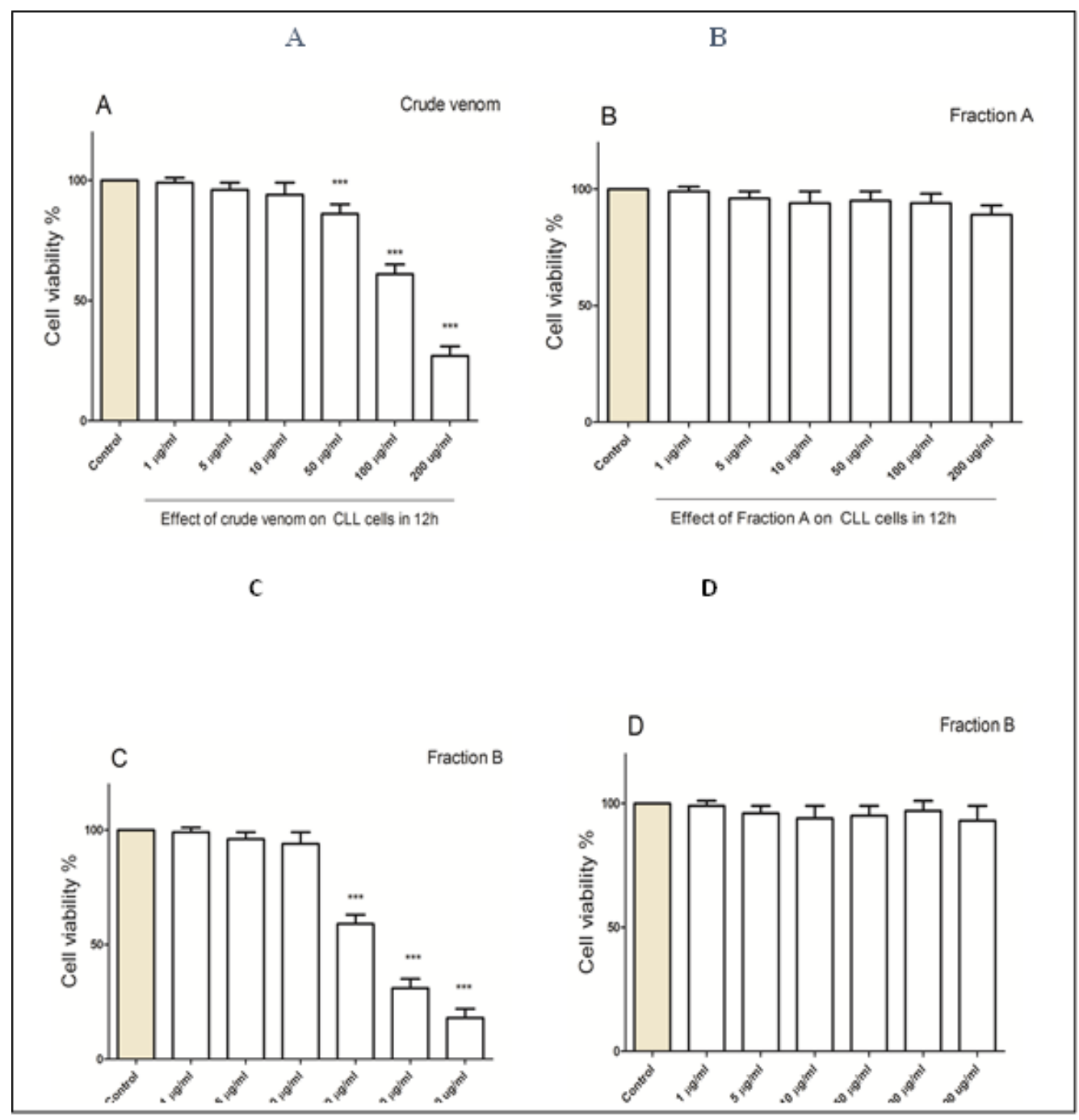

Figure 2. Cell Viability Assay. (A) Effect of crude venom on CLL B- lymphocytes in 12 hours, (B) effect of fraction A on CLL B- lymphocytes in 12 hours, (C) effect of fraction B on CLL B- lymphocytes in 12 hours, (D) effect of fraction $B$ on normal cells in 12 hours. All data were presented as the mean \pm S. $D(n=3)$. *** $p<0.001$ significantly different from the control group

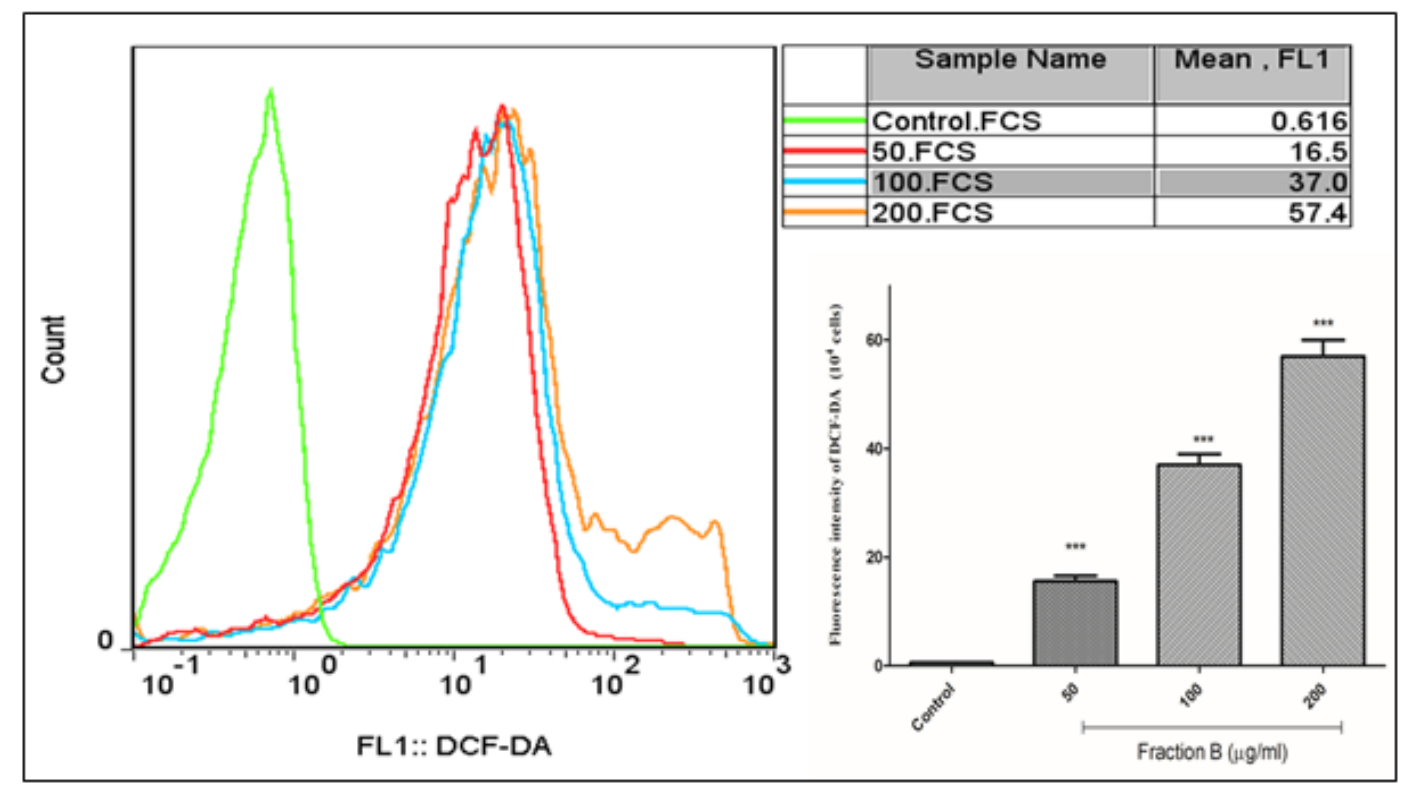

Figure 3. ROS Assay. Effect of fraction B (50, 100 and $200 \mu \mathrm{g} / \mathrm{ml})$ on ROS generation in CLL B- lymphocytes. All data were presented as the mean \pm S.D $(n=3)$. $* * * p<0.001$ significantly different from the control group. DCF-DA: Dichlorofluorescin Diacetate. FCS: Forward Channel Scatter. FL1: Fluorescence Channel 1. 


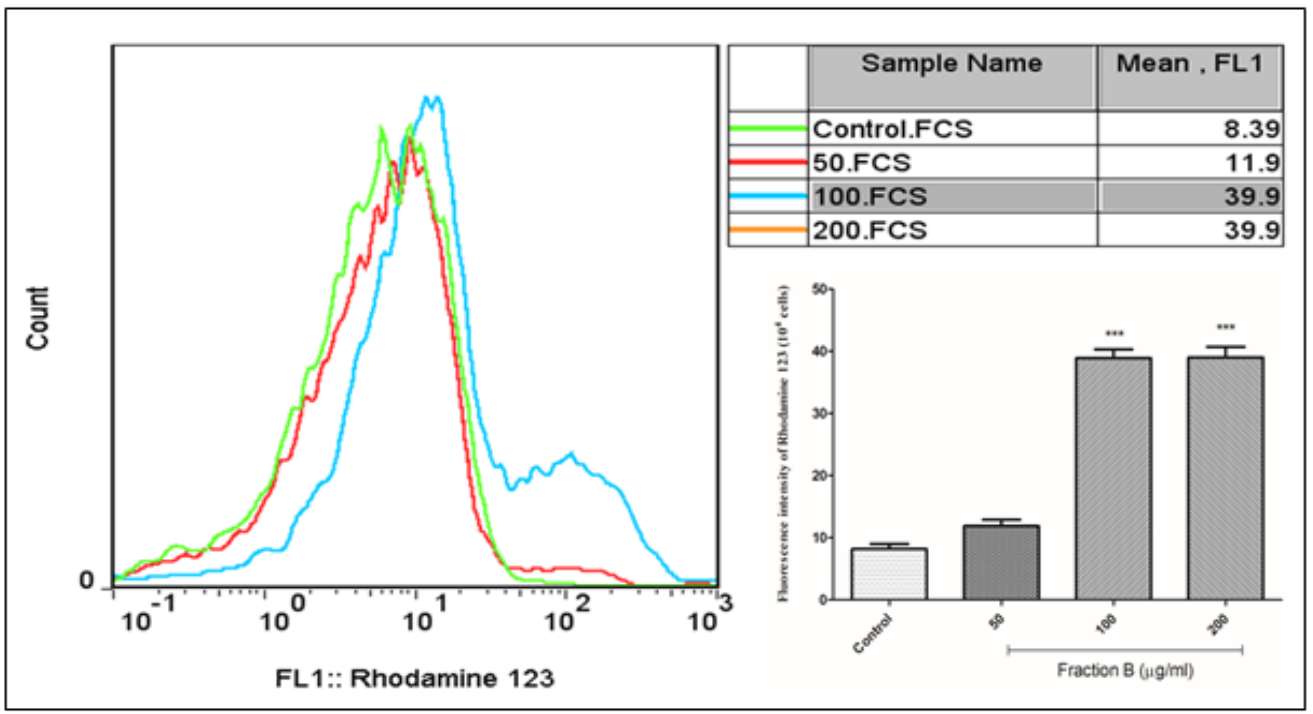

Figure 4. MMP Assay. Effect of fraction B (50, 100 and $200 \mu \mathrm{g} / \mathrm{ml})$ on MMP collapse in CLL B- lymphocytes. All data were presented as the mean \pm S.D $(n=3)$. *** $p<0.001$ significantly different from the control group. FCS, Forward Channel Scatter; FL1, Fluorescence Channel 1.

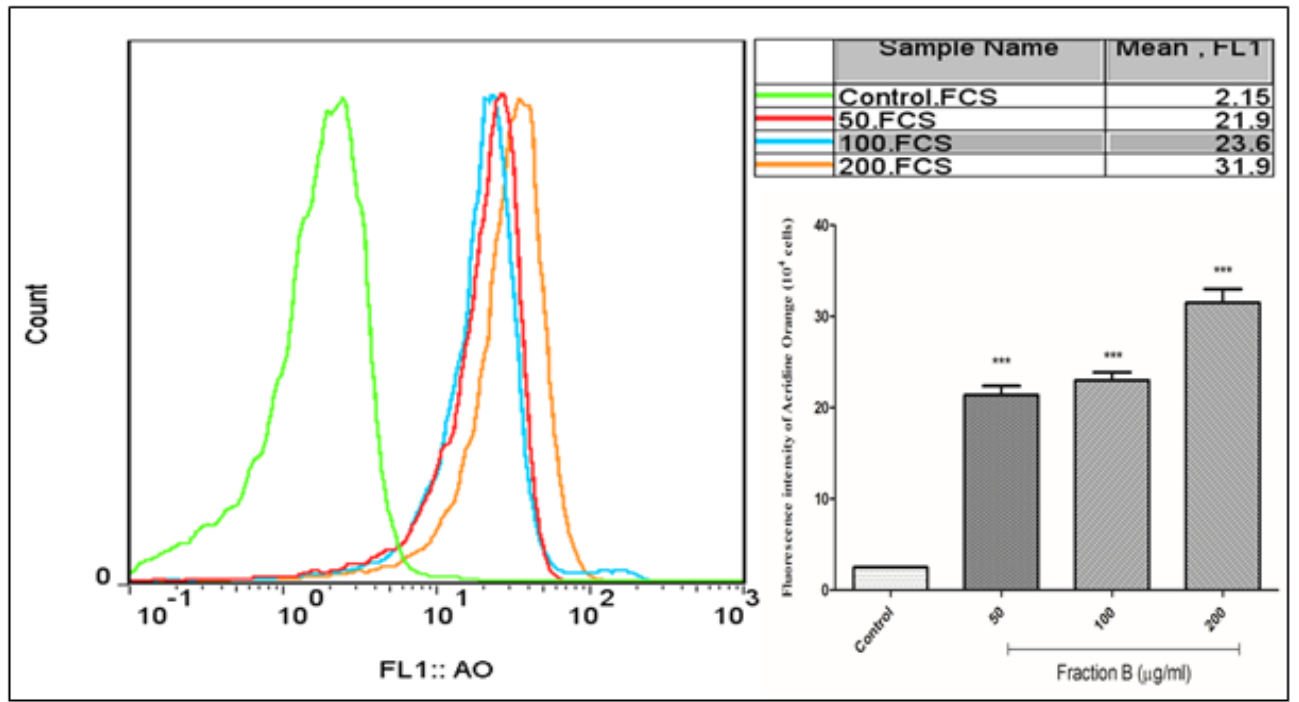

Figure 5. Lysosomal Damage Assay. Effect of fraction B (50, 100 and $200 \mu \mathrm{g} / \mathrm{ml})$ on lysosomal damage in CLL Blymphocytes. All data were presented as the mean \pm S.D $(n=3) . * * * p<0.001$ significantly different from the control group. FCS, Forward Channel Scatter; FL1, Fluorescence Channel 1; AO, Acridine Orange.

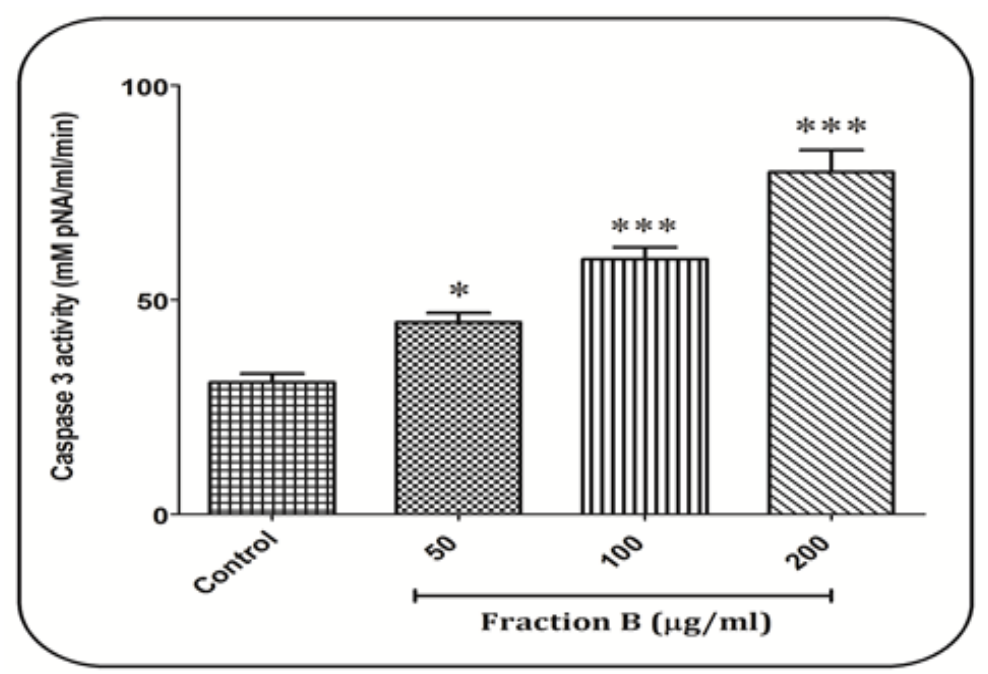

Figure 6. Caspase-3 Activity Assay. Effect of fraction B (50, 100 and $200 \mu \mathrm{g} / \mathrm{ml})$ on caspae-3 activity in CLL Blymphocytes. All data were presented as the mean $\pm \operatorname{S.D}(n=3)$. $* \mathrm{p}<0.05$ and $* * * \mathrm{p}<0.001$ significantly different from the control group. 
the discovery of new compounds with anti-cancer effects is essential (Aufschnaiter et al., 2020; Borojeni et al., 2020). Today, venoms isolated from animal sources are of great importance for the treatment of cancer. These venoms contain bioactive compounds such as proteins, salts, peptides and small molecules that have many pharmacological activities. Furthermore, isolated venoms have little toxic effects on normal cells and tissues and also can play a role in the induction of apoptosis signaling (Mirzaei et al., 2020; Roy and Bharadvaja, 2020).

Initially, the results showed that the crude venom and its fraction $\mathrm{B}$ had the ability to reduce the viability of CLL B-lymphocytes. These results are in agreement with previous studies that have shown that the use of venoms extracted from marine animals is associated with a decrease in the viability of various cancer cell lines (Catanesi et al., 2021; Rodrigo et al., 2021). Studies have shown that there is an inverse relationship between cell viability and apoptotic signaling ( $\mathrm{Li}$ et al., 2020; Wang et al., 2020). Therefore, it is possible that the decrease in cell viability by Conus textile in CLL cells is associated with activation of apoptotic signaling. Mitochondria have attracted a lot of attention as a therapeutic target. Mitochondria play important roles in physiological processes including the generation of ROS and apoptosis signaling (Fisher et al., 2020; He et al., 2020). Research has shown that an increased level of mitochondrial ROS in cancer cells is considered a therapeutic target. In addition, studies have shown that the level of free radicals in cancer cells is higher than in normal cells, and an excessive mitochondrial ROS can contribute to the kill of cancer cells. Therefore, compounds that can increase the level of ROS can be used to kill cancer cells.(Seydi et al., 2016; Seydi et al., 2018).

An association between ROS levels and apoptotic signaling has been reported. ROS can activate apoptotic signaling by releasing pro-apoptotic proteins (such as cytochrome c) from the mitochondria and activation of a caspase cascade (Simon et al., 2000; Li et al., 2003). The results of our study showed that fraction B obtained from Conus textile was able to increase the level of ROS in CLL. These results are in agreement with the results of previous studies that have shown that crude venom of Conus textile and other animal venoms have the ability to increase the level of ROS (Al-Asmari et al., 2018; Salimi et al., 2020).

The collapse in the MMP is one of the important events in apoptosis that can occur due to an increase in the level of ROS. It has been shown that collapse in the MMP may be associated with the release of pro-apoptotic proteins as well as caspase cascade activation (Seydi et al., 2016; Seydi et al., 2018). The increase in the level of ROS due to fraction B of Conus textile in CLL B-lymphocytes has been associated with the collapse of MMP. These results from this venom have been proven in our previous study on U87MG human glioma cells (Salimi et al., 2020). Lysosomes is considered as another source of ROS and can also play a role in apoptosis signaling (Zhang et al., 2009). The results showed that fraction $\mathrm{B}$ in a dose-dependent pattern caused damage to the lysosomal membrane in CLL B-lymphocytes. Apoptosis is a type of cell death and the mitochondrial pathway is one of the most important pathways involved in apoptosis signaling. Caspase 3 plays an important role in apoptosis as a final mediator (Seydi et al., 2016; Zhou et al., 2021). The result showed that fraction B selectively could induce activation of caspase-3 via mitochondrial pathway in CLL B-lymphocytes. These results are in agreement with our previous study (Salimi et al., 2020).

Our findings in this study suggest that fraction B from crude venom of Conus textile has been able to increase the level of ROS in CLL cells by acting on mitochondria and lysosomes. Subsequently, ROS was able to activate caspase- 3 by acting on MMP and the release of pro-apoptotic proteins. Accordingly, Conus textile can be a promising source for anticancer drug candidates.

\section{Author Contribution Statement}

Ahmad Salimi and Enayatollah Seydi contributed to this research in in carrying out the experiments, analyzing the data and writing the paper. Shayan Salehian contributed to this research in carrying out the experiments and performing statistical analysis as the thesis student. Vahed Adhami, Amir Vazirizadeh and Seyyed Hossein Sajjadi Alehashem contributed in this research in carrying out some experiments. Jalal Pourahmad contributed to this research in formulating the research question (s), designing the study, carrying it out as thesis supervisor, analyzing the data, and writing paper. Akram Aboutorabi contributed to this research in designing part of the study and carrying it out as thesis co-supervisor.

\section{Acknowledgements}

\section{Funding statement}

The data provided in this manuscript were extracted from the PharmD thesis of Dr. Shayan Salehian. Experimental work was funded by Shahid Beheshti University of Medical Sciences, Tehran, Iran.

\section{Ethics approval}

All investigations were done according to the standard protocols approved by the ethic of Shahid Beheshti University of Medical Sciences, Tehran, Iran (ethic number: IR.SBMU.PHARMACY.REC.1398.315).

\section{Conflict of Interests}

The authors declare that they have no conflict of interest.

\section{References}

Abdel-Rahman MA, Abdel-Nabi IM, El-Naggar MS, et al (2013). Conus vexillum venom induces oxidative stress in Ehrlich's ascites carcinoma cells: an insight into the mechanism of induction. $J$ Venom Anim Toxins Incl Trop Dis, 19.

Al-Asmari AK, Riyasdeen A, Islam M (2018). Scorpion venom causes apoptosis by increasing reactive oxygen species and cell cycle arrest in MDA-MB-231 and HCT-8 cancer cell lines. J Evid Based Integr Med, 23, 2156587217751796.

Alburae NA, Mohammed AE (2020). Antiproliferative effect of 
the Red Sea cone snail, Conus geographus. Trop J Pharm Res, 19, 577-81.

Aufschnaiter A, Kohler V, Khalifa S, et al (2020). Apitoxin and its components against cancer, neurodegeneration and rheumatoid arthritis: Limitations and possibilities. Toxins, 12, 66.

Baracca A, Sgarbi G, Solaini G, et al (2003). Rhodamine 123 as a probe of mitochondrial membrane potential: evaluation of proton flux through F0 during ATP synthesis. Biochim Biophys Acta Bioenerg, 1606, 137-46.

Borojeni SK, Zolfagharian H, Babaie M, et al (2020). Cytotoxic effect of Bee (A. mellifera) Venom on cancer cell lines. J Pharmacopuncture, 23, 212.

Brunk U, Zhang H, Roberg K, et al (1995). Lethal hydrogen peroxide toxicity involves lysosomal iron-catalyzed reactions with membrane damage. Redox Rep, 1, 267-77.

Catanesi M, Caioni G, Castelli V, et al (2021). Benefits under the Sea: The role of Marine compounds in neurodegenerative disorders. Mar Drugs, 19, 24.

Chatterjee B (2018). Animal Venoms have potential to treat cancer. Curr Top Med Chem, 18, 2555-66.

Fisher JJ, Bartho LA, Perkins AV, et al (2020). Placental mitochondria and reactive oxygen species in the physiology and pathophysiology of pregnancy. Clin Exp Pharmacol Physiol, 47, 176-84.

Fu Y, Li C, Dong S, et al (2018). Discovery methodology of novel conotoxins from Conus species. Mar Drugs, 16, 417.

Gao B, Peng C, Lin B, et al (2017a). Screening and validation of highly-efficient insecticidal conotoxins from a transcriptomebased dataset of chinese tubular cone snail. Toxins, 9, 214.

Gao B, Peng C, Yang J, et al (2017b). Cone snails: A big store of conotoxins for novel drug discovery. Toxins, 9, 397.

He Y, Fang X, Shi J, et al (2020). Apigenin attenuates pulmonary hypertension by inducing mitochondria-dependent apoptosis of PASMCs via inhibiting the hypoxia inducible factor $1 \alpha-$ KV1. 5 channel pathway. Chem Biol Interact, 317, 108942 .

Jimenez PC, Wilke DV, Branco PC, et al (2020). Enriching cancer pharmacology with drugs of marine origin. $\mathrm{Br} J$ Pharmacol, 177, 3-27.

Li N, Pan J, Liu W, et al (2020). MicroRNA-15a-5p serves as a potential biomarker and regulates the viability and apoptosis of hippocampus neuron in children with temporal lobe epilepsy. Diagn Pathol, 15, 1-7.

Li N, Ragheb K, Lawler G, et al (2003). Mitochondrial complex I inhibitor rotenone induces apoptosis through enhancing mitochondrial reactive oxygen species production. J Biol Chem, 278, 8516-25.

Mirzaei S, Fekri HS, Hashemi F, et al (2020). Venom peptides in cancer therapy: An updated review on cellular and molecular aspects. Pharmacol Res, 105327.

Oroz-Parra I, Álvarez-Delgado C, Cervantes-Luevano K, et al (2020). Proapoptotic index evaluation of two synthetic peptides derived from the Coneshell Californiconus californicus in lung cancercell 1.ine H1299. Mar Drugs, 18, 10 .

Rigby AC, Lucas-Meunier E, Kalume DE, et al (1999). A conotoxin from Conus textile with unusual posttranslational modifications reduces presynaptic $\mathrm{Ca} 2+$ influx. Proc Natl Acad Sci U S A, 96, 5758-63.

Rodrigo AP, Mendes VM, Manadas B, et al (2021). Specific antiproliferative properties of proteinaceous toxin secretions from the Marine Annelid Eulalia sp. onto ovarian cancer cells. Mar Drugs, 19, 31.

Roy A, Bharadvaja N (2020). Venom-derived bioactive compounds as potential anticancer agents: a review. Int $J$ Pept Res Ther, 2020, 1-19.
Ruiz-Torres V, Encinar JA, Herranz-López M, et al (2017). An updated review on marine anticancer compounds: The use of virtual screening for the discovery of small-molecule cancer drugs. Molecules, 22, 1037.

Salimi A, Rahimitabar N, Vazirizadeh A, et al (2020). Persian Gulf Snail Crude Venom (Conus textile): A potential source of anti-cancer therapeutic agents for glioblastoma through mitochondrial-mediated apoptosis. Asian Pac J Cancer Prev, 21, 49-57.

Salimi A, Roudkenar MH, Sadeghi L, et al (2015). Ellagic acid, a polyphenolic compound, selectively induces ROS-mediated apoptosis in cancerous B-lymphocytes of CLL patients by directly targeting mitochondria. Redox Biol, 6, 461-71.

Seydi E, R Rasekh H, Salimi A, et al (2016). Selective toxicity of apigenin on cancerous hepatocytes by directly targeting their mitochondria. Anti-Cancer Agents Med Chem, 16, 1576-86.

Seydi E, Salimi A, Rasekh HR, et al (2018). Selective cytotoxicity of luteolin and kaempferol on cancerous hepatocytes obtained from rat model of hepatocellular carcinoma: involvement of ROS-mediated mitochondrial targeting. Nutr Cancer, 70, 594-604.

Simon H-U, Haj-Yehia A, Levi-Schaffer F (2000). Role of reactive oxygen species (ROS) in apoptosis induction. Apoptosis, 5, 415-8.

Sukmarini L (2021). Drug Development from Peptide-derived Marine Natural Products. IOP Conf Ser Mater Sci Eng, IOP Publishing, 012063.

Sun Z, Zhangsun M, Dong S, et al (2020). Differential expression of nicotine acetylcholine receptors associates with human breast cancer and mediates antitumor activity of $\alpha \mathrm{O}$ Conotoxin GeXIVA. Mar Drugs, 18, 61.

Teoh CS, Goh AS (2021). Relapsed chronic lymphocytic leukaemia with concomitant extensive chronic Graft versus host disease after allogeneic haematopoietic stem cell transplantation successfully treated with oral venetoclax. Case Rep Transplant, 2021.

Wali AF, Majid S, Rasool S, et al (2019). Natural products against cancer: review on phytochemicals from marine sources in preventing cancer. Saudi Pharm J, 27, 767-77.

Wang S, Zhao G, Zhao S, et al (2020). The effects of interleukin-33 (IL-33) on osteosarcoma cell viability, apoptosis, and epithelial-mesenchymal transition are mediated through the PI3K/AKT pathway. Med Sci Monit, 26, e920766-1.

Zhang H, Zhong C, Shi L, et al (2009). Granulysin induces cathepsin B release from lysosomes of target tumor cells to attack mitochondria through processing of bid leading to Necroptosis. J Immunol, 182, 6993-7000.

Zhang Q, Zhao X-H, Wang Z-J (2008). Flavones and flavonols exert cytotoxic effects on a human oesophageal adenocarcinoma cell line (OE33) by causing G2/M arrest and inducing apoptosis. Food Chem Toxicol, 46, 2042-53.

Zhou L, Wang S, Cao L, et al (2021). Lead acetate induces apoptosis in Leydig cells by activating PPAR $\gamma /$ caspase-3/ PARP pathway. Int J Environ Health Res, 31, 34-44.

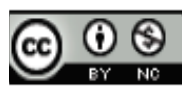

This work is licensed under a Creative Commons AttributionNon Commercial 4.0 International License. 